\title{
3-D SYNTHETIC APERTURE RADAR INTERFEROMETRY PHASE UNWRAPPING USING EXTENDED KALMAN FILTERS
}

\author{
B. Osmanoglu ${ }^{1,2}$, S. Wdowinski², T. H. Dixon ${ }^{3}$ \\ 1.USRA - NASA GSFC, 8800 Greenbelt Rd., Greenbelt, MD 20771 \\ 2. University of Miami - RSMAS, 4600 Rickenbacker CSWY, Miami, FL 33149 \\ 3. University of South Florida, 4202 E. Fowler Ave, Tampa, FL 33620
}

\begin{abstract}
:
Synthetic Aperture Radar Interferometry (InSAR) observations allow researchers to map elevations, analyze surface deformation, and even detect ground water level changes from satellites orbiting the Earth. The InSAR phase measurements are inherently wrapped between 0 and $2 \pi$. For most physical interpretation methods the phase measurements have to be unwrapped to reveal the full scale of the observations. The unwrapping of multi-dimensional phase data is still a field of active research and here we present an algorithm using an Extended Kalman Filter (EKF).
\end{abstract}

The current implementation of our EKF algorithm utilizes a piecewise linear approximation in space and a simple model in the third dimension (e.g. time). The algorithm starts from wrapped, unfiltered interferograms and filters and unwraps the results at the same time solving for a common topography or deformation rate, starting from the highest quality point in the coherent area and proceeding to unwrap highest quality neighbors. The highest quality neighbors are determined according to the Fisher's Distance, which is a phase quality measure similar to the more commonly used phase derivative variance, but also includes the interferogram coherence. In this presentation we demonstrate the effectiveness of our algorithm for the applications of DEM generation and deformation rate analysis using real data.

\section{INTRODUCTION}

EKF operates by making a prediction based on the current state, and verifying the prediction by incoming measurements in the control step. The prediction step combines information from previously unwrapped neighbors which is verified against the interferograms in the control step. In the smoothing step, the same EKF updates the result for pixels with 4 neighbors. Once the entire data is unwrapped modified Frankot-Chellappa (MFC) algorithm is utilized to estimate a path independent solution using only the gradients from EKF. The path independent solution is compared against the EKF result.

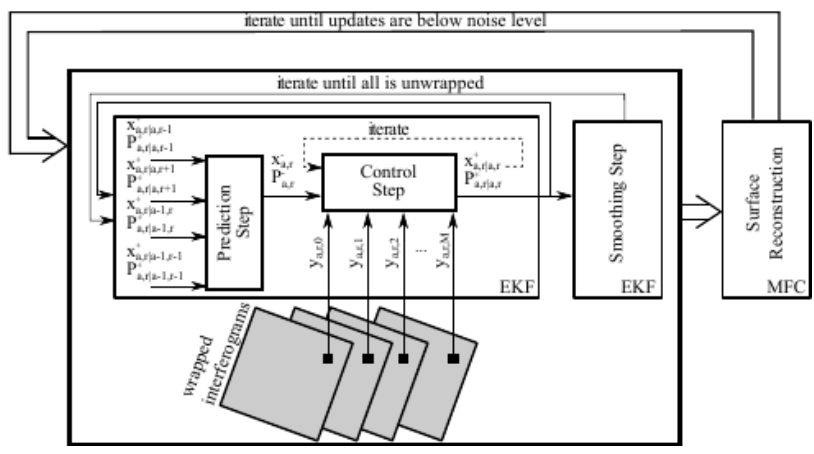

Figure 1: Box diagram for the proposed method. The figure displays the EKF variables: $x$ (EKF state), $P$ (Error Covariance), $y$ (interferometric observations), a (azimuth), $r$ (range).

The proposed algorithm is an improved version of our earlier work (Osmanoglu et al. 2013). Presented results are compared to those of the conventional DEM generation method, namely a weighted average of individually unwrapped interferograms using the Statistical Cost, Network-Flow Algorithm for Phase Unwrapping (SNAPHU), as well as the results from 3-D Maximum-Likelihood Algorithm (3DMLE) (Costantini, 1998; Eineder and Adam, 2005; Crosetto, 2002; Chen and Zebker,
2001; Chen, 2001). For the deformation analysis the EKF results are compared to PSI and SBAS.

\section{DEM GENERATION}

To demonstrate unwrapping of phase induced by topography from a multi-baseline dataset we used four TerraSAR-X interferograms over El Paso - Juarez (US-Mexico border). Interferogram selection was made such that the temporal separation was small and perpendicular baselines were favorable for topography reconstruction.

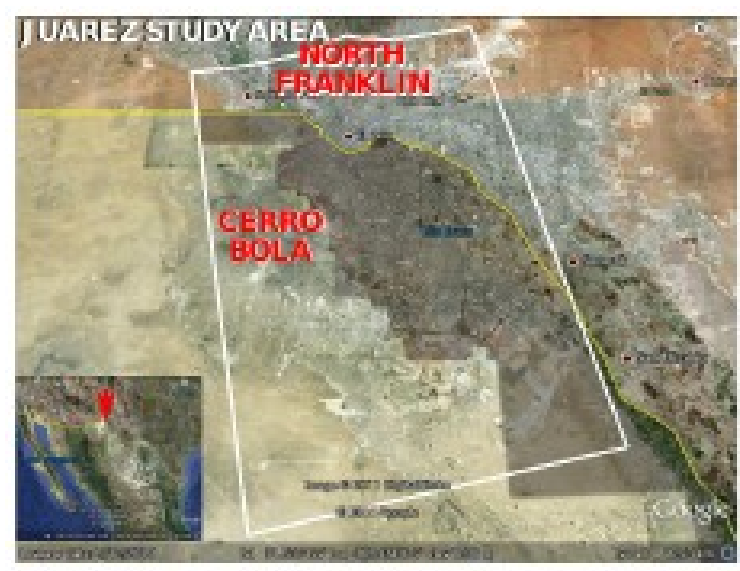

Figure 2: Study area. North Franklin and Cerro Bola mountains are shown with red labels. The inset provides relative location. 
100904-100221 100108-091228 100531-100520 100130-10022
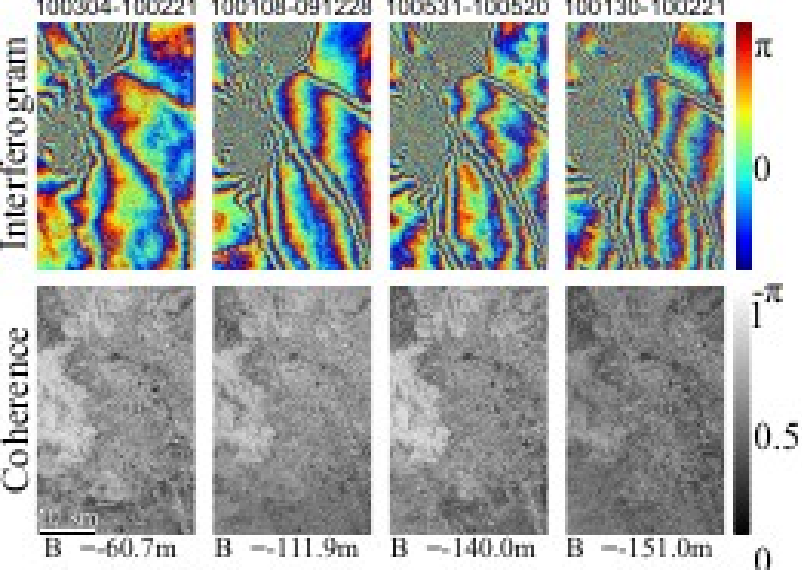

Figure 3: Interferograms and coherence values. The perpendicular baselines increase from left to right. Top labels provide the master and slave dates.
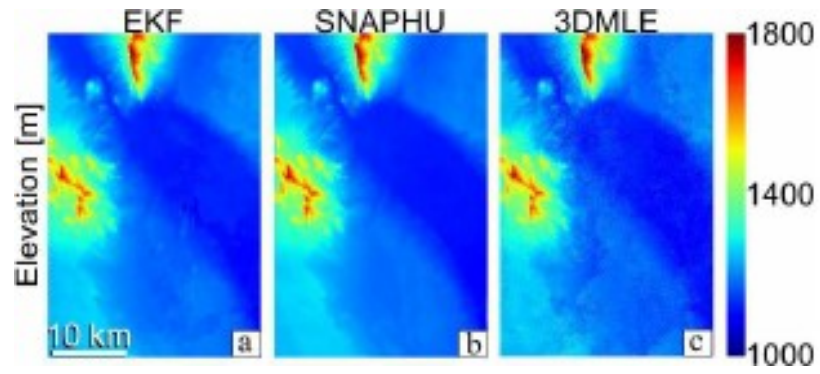

Figure 4: Estimated elevations using: a) EKF b) unwrapped using SNAPHU and weighted averaged c) unwrapped using 3D-Maximum Likelihood Estimator.

Results are compared to a NED (National Elevation Dataset) $1 / 9 \operatorname{arcsec}(\sim 3 \mathrm{~m})$ DEM. EKF provides the best fit. Residuals and misfit is shown in Figure 5. The misfit is a unitless measure of fit, defined as:

$$
\sqrt{\left\langle\frac{[\hat{z}-z]^{2}}{\sigma_{z}^{2}}\right\rangle}
$$

where $\mathrm{z}$ denotes elevation and $\sigma$ denotes expected standard deviation of the measurement.

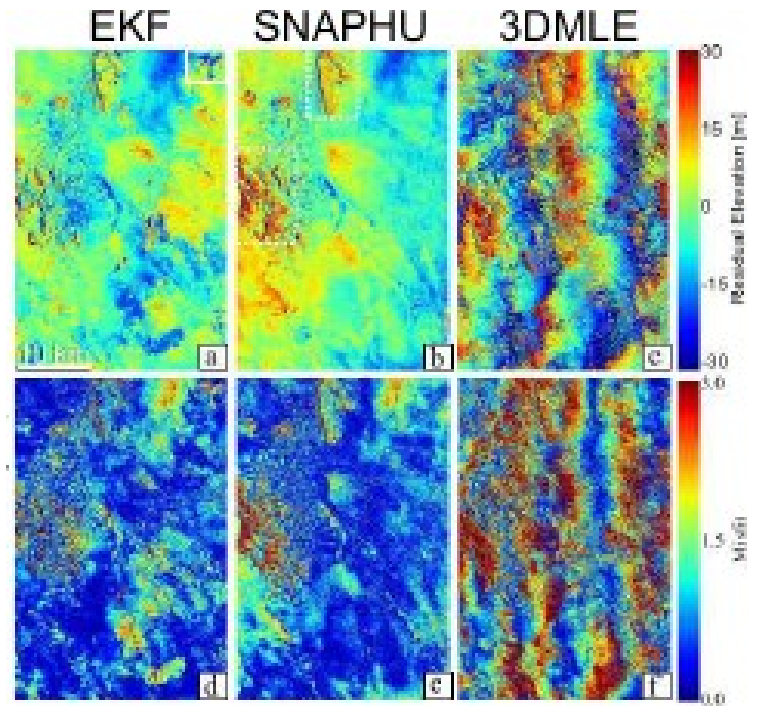

Figure 5: Residuals are shown through a,b, and c, while the misfit is shown in d,e and f. For DEM a nalysis we did not utilize a surface reconstruction or use any global iterations. Therefore minor error propagation is visible in a. EKF performs better over mountainous areas highlighted in $b$. 3DMLE does not resolve the phase ambiguity correctly in c.

\section{DEFORMATION ANALYSIS}

23 Envisat ASAR images were used for the analysis of Mexico City subsidence. The PSI analysis used all the scenes, while the EKF, SBAS and NSBAS analysis used the interferograms shown in Figure 6.

Some of the interferograms with long temporal baselines are unwrapped wrong due to rapid subsidence signal. Wrong spatial unwrapping causes the SBAS and NSBAS algorithms to underestimate the deformation.

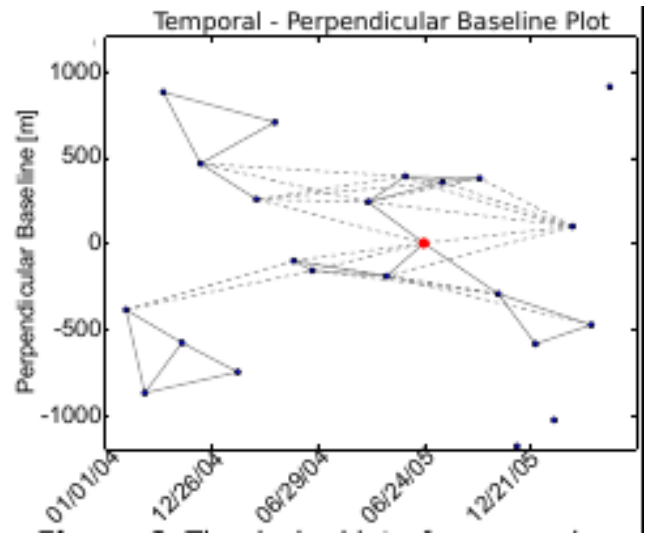

Figure 6: The dashed interferograms have temporal baselines of more than 175 days. PSI master image is shown in red.

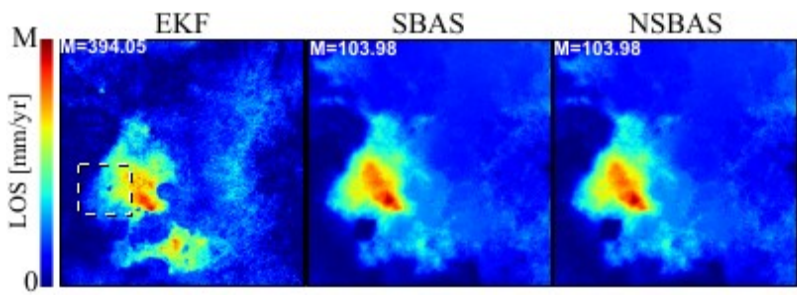

Figure 7: Comparison of EKF, SBAS and NSBAS results. All algorithms capture the deformation pattern however the deformation rates are lower for SBAS and NSBAS. M denotes the maximum rate.

Results from EKF are compared to the results from PSI from an earlier work (Osmanoglu et al., 2011). The results match rather well.

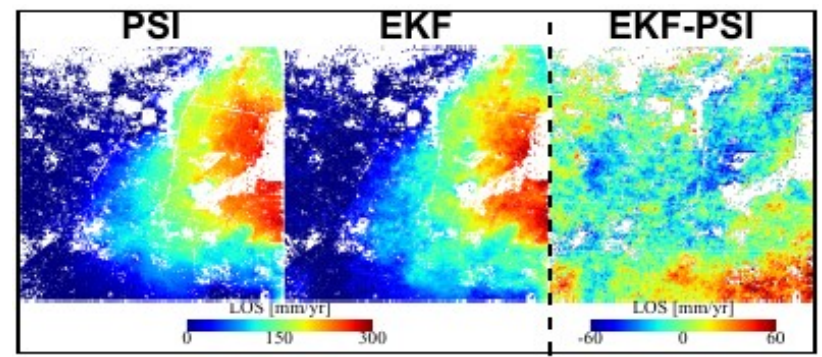


Figure 8: Comparison of PSI and EKF results. The overall trend is correctly matched with EKF, and there are differences up to $60 \mathrm{~mm} / \mathrm{yr}$. Coverage of the PSI analysis is shown in Figure 7 with dashes lines.

\section{CONCLUSION}

We developed a new unwrapping method based on extended Kalman filtering to estimate topography and deformation time series from SAR images. Time series is generated using both spatial and temporal relations in the data set. Obtained results with real data show good agreement with other measurements. Future improvements will incorporate a non-linear deformation analysis.

\section{Acknowledgements}

The authors would like to thank NASA for the NESSF Fellowship grant 09-Earth-09R-61 and research grant NNX12AK23G. We thank ESA for providing the Envisat imagery through GEO, and DLR for the TSX imagery through HYD 0655.

\section{REFERENCES}

M. Costantini, "A novel phase unwrapping method based on network programming," Geoscience and Remote Sensing, IEEE Transactions on, vol. 36, no. 3, pp. 813-821, May 1998.

M. Eineder and N. Adam, "A maximum-likelihood estimator to simultaneously unwrap, geocode, and fuse sar interferograms from different viewing geometries into one digital elevation model," Geoscience and Remote Sensing, IEEE Transactions on, vol. 43, no. 1, pp. 24-36, 2005.

M. Crosetto, "Calibration And Validation of SAR Interferometry for DEM Generation," ISPRS Journal of Photogrammetry and Remote Sensing, vol. 57, no. 3, pp. 213-227, 2002.

C. W. Chen and H. A. Zebker, "Two-dimensional phase unwrapping with use of statistical models for cost functions in nonlinear optimization," J. Opt. Soc. Am. A, vol. 18, no. 2, pp. 338-351, 2001.

C. W. Chen, "Statistical-cost network-flow approaches to two-dimensional phase unwrapping for radar interferometry," Ph.D. Dissertation, Stanford University. Dept. of Electrical Engineering., Jun. 2001

B. Osmanoglu, T. H. Dixon, S. Wdowinski, and E. Cabral-Cano, "On the importance of Path for Phase Unwrapping in Synthetic Aperture Radar Interferometry," Applied Optics, vol. 50, no. 19, pp. 3205-3220, 2011.

B. Osmanoglu, T. Dixon and S. Wdowinski, "3-D phase unwrapping for satellite radar interferometry, I: DEM generation", IEEE Trans. on Geoscience and Remote Sensing, doi: 10.1109/TGRS.2013.2247043 